\title{
Editorial
}

\section{Advances in electromagnetic applications and communications}

\author{
Erick Reyes-Vera ${ }^{1}$, David E. Senior ${ }^{2}$, José Martin Luna-Rivera ${ }^{3}$ y Francisco Lopez ${ }^{4}$
}

${ }^{1}$ Department of Electronic and Telecommunications, Instituto Tecnológico Metropolitano, Medellín- Colombia, erickreyes@itm.edu.co

2 Wireless Group, Anaren, a TTM Technologies Company, East Syracuse-USA, david.senior@ttm.com

${ }^{3}$ Universidad Autónoma de San Luis Potosí (UASLP), Faculty of Science, San Luis PotosíMéxico,mlr@fciencias.uaslp.mx

${ }^{4}$ Department of Electronic and Telecommunications, Instituto Tecnológico Metropolitano, Medellín- Colombia, franciscolopez@itm.edu.co

Modern communication systems have traditionally exploited three regions of the electromagnetic spectrum: radio waves, infrared and visible. Evolution in these ranges is always accompanied by the appropriation of new electromagnetic phenomena to build devices with better characteristics. In these three regions, great advances have been made in recent years. For that reason, in this issue, we call for papers concerning the biggest challenges these technologies may face in the years to come.

The first region to be explored is radio waves, in which wireless communications have seen great developments. Antennas, for example, have always been fundamental parts of wireless communication systems, from the simplest AM radio transceiver to the most complicated smart phone, mm-wave imaging device, or wireless power transfer solution. A great deal of industry efforts focuses on new and future applications connected in the so-called Internet of Things (IoT), such as autonomous cars, smart cities, wearable devices, industrial internet, smart homes and connected healthcare, among others. Such applications would require a massive amount of data, faster speeds and, of course, complicated antenna systems working in a wide range of frequency bands [1]. According to Gartner, there will be nearly 21 billion connected IoT devices by 2020, while Ericsson predicts a global mobile traffic of more than 60 ExaBytes (EB) per month by 2022 [2]. From the point of view of mobile technologies, 5G, the next-generation mobile network, is envisioned to be the solution that will enable all these connected applications by allowing ubiquitous, high-speed, reliable and high-volume Internet connectivity between connected devices from anywhere [3]. Due to the expected massive amount of data and high connection speeds on $5 \mathrm{G}$ networks, the $5 \mathrm{G}$ New Radio (NR) recently released standard has proposed millimeter-wave frequency bands to be used as part of the solution (for example, from $24 / 28 / 39 \mathrm{GHz}$ up to $86 \mathrm{GHz}$ ) [3]. Moreover, the proposed use of Massive Multiple Input/Multiple Output (MIMO), which consists of groups of antennas at the transmitter and receiver, would provide better mobile capacity, coverage, reliability and spectrum efficiency [3]. With the extremely short wavelengths millimeter-wave signals offer (for example, $10.7 \mathrm{~mm}$ at $28 \mathrm{GHz}$, and $7.7 \mathrm{~mm}$ at $39 \mathrm{GHz}$ ), consumer electronic products would have physically integrated small adaptive antenna arrays, either On-Chip or In-Package, that offer high gain, low cost, and flexibility [4]. This is a unique opportunity for antenna engineers to develop innovative, low-cost, fully-integrated and small solutions for next-generation devices. 
It is therefore broadly accepted that traditional antenna technology needs a leap forward in order to create integrated antenna solutions for $5 \mathrm{G}$ and other advanced new and future applications. And that step is already being taken with a lot of new developments, approaches and materials whose ultimate goal is to improve antenna performance. From 3D printed antennas based on metal plated polymer materials for satellite communications, to miniaturized metamaterial-based antennas allowing beam steering without phase shifters, high-performance electrically small fractal antennas, mm-wave phased arrays on low temperature cofired ceramic (LTCC), and plasma-silicon devices for efficient beamforming, the future looks promising [5]. In this line, for example, Qualcomm recently demonstrated the first mm-wave $5 \mathrm{G}$ antenna module for smartphones, which is a phased array of four antennas small enough to fit into smartphones and other mobile devices [6]. So, the future is here, and more research is necessary to overcome all the design and fabrication challenges $\mathrm{mm}$-wave antennas impose, such as the achievement of high radiation efficiency, low gain fluctuation, high isolation between individual elements, efficient beam steering, lower fabrication tolerances, and new characterization methods.

In the infrared region, optical fibers are the preferred transmission channel. Such fibers have been extensively used in metropolitan networks for long-haul terrestrial and transoceanic links because, compared to other technologies, they offer several advantages, such as low-loss, large bandwidth, and electromagnetic immunity, among others. Since 2000, the traffic in optical communications networks has grown exponentially, nearly $50 \%$ to $60 \%$ per year, as confirmed by Bell Labs [7]. These data are associated with the ever-growing number of users and devices connected to the Internet around the world. In order to satisfy the great demand for information and increase transmission rates, several technological breakthroughs were necessary, e.g. low-loss Single Mode Fibers (SMF), Erbium-Doped Fiber Amplifiers (EDFAs), Wavelength Division Multiplexing (WDM), Polarization Division Multiplexing (PDM) and high-spectral-efficiency coding through a Digital Signal Processor (DSP) that enables coherent transmission [8]. In addition, the transmission of signals using each wavelength as an independent channel, known as WDM, is the dominant technology; which was developed for increasing fiber links capacities. In addition to such improvement in channel capacity, WDM optical networks also bring the advantages of easy routing and switching based on wavelength schemes [9]. Currently, WDM coherent optical communication systems have already depleted all degrees of freedom, namely, frequency, quadrature, and polarization in single-mode fiber. As a result, space is the only extra dimension that can be exploited in the future of optical fiber communication systems and, in order to use this degree of freedom, Space-Division Multiplexing (SDM) has been proposed [8]. In SDM systems, independent data streams can be transmitted in parallel spatial channels. SDM can be implemented in two different ways: first, with Multi-Core Fibers (MCF) using each core as an independent channel for sending information [8], [10]; and second, with Modal Division Multiplexing (MDM), which sends coded information through different propagation modes of a multimode fiber [11]. Mode Multiplexers (MUX) and Demultiplexers (DEMUX) are critical components for SDM transmission. They transform signals from parallel SMFs to SDM signals that contain the superposition of different modes. Each MUX has two components: mode-converter and combiner. Therefore, to correctly implement this technique, new devices allowing to convert and control propagated modes in an optical fiber must be designed. In turn, Digital Signal Processing (DSP) should be implemented in order to compensate for the linear channel impairments generated as consequence of the coupling between different propagated modes. As a result, in the future, DSP systems could play an important role in optic communications by guaranteeing the quality and stability of the information, which makes this one of the biggest challenges in the field.

[10] TecnoLógicas, ISSN-p 0123-7799 / ISSN-e 2256-5337, Vol. 21, No. 43, sep-dic de 2018, pp. 9-13 
An interesting alternative to fiber optic communications is the use of free space optical communications (FSO)[12]. which has drawn great attention as an substitute of wireless communications. In FSO, the information is sent using laser or LED sources, such as in fiber-optic communications. However, in this case, light propagates through the air and, currently, the technology can operate only from $780 \mathrm{~nm}$ to $1600 \mathrm{~nm}$. A specific case of this type of technology is Visible Light Communication (VLC) [12], [13]. VLC is a type of Optical Wireless Communications Technology, which encompasses areas as diverse as Communications in high-speed FSO, underwater communication systems, or short-distance and low-speed systems. This field of application has attracted the attention of several standardization committees; among them, we can highlight IEEE 802.15.7, which has focused on low/medium-speed systems, leaving high bandwidth systems to IEEE 802.15.13. In addition, as we are dealing with systems that generally work in domestic environments with the presence of humans, it is necessary to introduce additional restrictions such as eye safety, ergonomics, and comfort measures (e.g. dimming options, avoiding flickering, or adjustable color temperature). Another critical aspect in this type of systems is energy efficiency because one of the reasons that led to the success of lighting systems based on solid-state lamps is their drastic reduction of consumption compared to other alternatives, such as incandescent, fluorescent, ballast or halogen lamps.

Nowadays, solid state lamps are universally present in our lives, both as a lighting medium and in ubiquitous systems such as smartphones. The possibility of using OCC (Optical Camera Communications) systems in this type of devices opens a new field of high-security communications that can be employed in different areas: real time location, identity verification, key sharing in areas such as verification of secure payments, electronic commerce, or video games. Additionally, in the fields of communications between vehicles (V2V), or from these with the infrastructures of roads, tunnels or traffic lights (I2V), this technology offers solutions for the transmission of vehicle diagnosis as well as intelligent driving systems. Moreover, the recent adoption in the United States of a regulation that forces manufacturers to equip new vehicles with a front camera offers a window of opportunity for new technological developments.

The call for papers on the topics above prompted a very positive response from researches around the world. As consequence, we received 17 submissions from Colombia, Argentina, Mexico, and Poland. We were delighted to witness the massive reaction of by our colleagues, who reported interesting research results related with different fields: networks defined by software, visible light communications, wireless sensors, mobile communications, electro-optic devices, radio over fiber, optic communications, and RF devices. Afterward, a careful and rigorous peer review process of the papers was carried out with the help of expert colleagues in related fields, who voluntarily examined these works (our goal is to have at least two reviewers per paper). After the review process, we calculated the acceptance rate: $17.65 \%$. Besides, four other manuscripts required major changes and continue with the editorial process to be included in future issues. These statistics show the high quality of the works published in this issue, in which we present the following three papers on advances in electromagnetic applications and communications.

In the first work of this issue, the authors propose the Bidirectional single-sideband transmission of millimeter waves over fiber for $5 \mathrm{G}$ mobile networks. Their manuscript presents an interesting architecture for bidirectional transmission of millimeter waves over optical fiber using a double single-side-band modulation in order to generate an optical subcarrier for the downlink transmission and a second subcarrier for the transport of the uplink. The optical subcarriers were generated using single-sideband modulation in a Mach-Zehnder electro-op- 
tical modulator. Finally, they validated the performance of the proposed architecture by analyzing typical indicators such as BER and Error Vector Magnitude in both the downlinks and the uplinks.

Next, the article titled Quality of service evaluation based on network slicing for softwaredefined 5G systems reveals the possibility of assessing the quality of services by adopting the network slicing approach for 5G networks and using a software-defined network environment. Floodlight, the open-source controller implemented in that work, was responsible for making bandwidth allocation decisions. The main contribution of said manuscript is describing the capacity of this type of solutions to control bandwidth resources, which allows to guarantee latency and reliability values. Therefore, they enable to reduce the loss rate by $10 \%$ in a congested network and assure delays under $700 \mathrm{~ms}$ for typical commercial applications.

Finally, First principles calculations of the electronic and dielectric properties of $\lambda$-Ta $\mathrm{Ta}_{2} \mathrm{O}_{5}$ delves into some physical properties of an orthorhombic $\lambda$-Tantalum pentoxide model without oxygen vacancies by applying the density functional perturbation theory. The authors obtained a set of interesting results related to the prediction of the crystalline structure of the model, the structure of electronic bands, dielectric tensor components, and optical response (refractive index, extinction coefficient, and transmittance). This material is of great interest for the development of modern electromagnetic devices for microwave applications such as antennas, filters, or resonators where the material plays an important role in performance and size.

The other seven papers published in this issue were submitted following the regular call for papers, which is permanently open. At the end of the process, a total of 37 manuscripts were evaluated and 10 of them (27\%) were accepted to be part of this issue.

\section{REFERENCES}

[1] M. H. Miraz, M. Ali, P. S. Excell, and R. Picking, "A review on Internet of Things (IoT), Internet of Everything (IoE) and Internet of Nano Things (IoNT)," in 2015 Internet Technologies and Applications (ITA), 2015, pp. 219-224.

[2] Ericsson, "The Ericsson Mobility Report," 2018. [Online]. Available: https://www.ericsson.com/en/mobility-report.

[3] 3GPP A Global Initiative, "Release 15," 2018. [Online]. Available: http://www.3gpp.org/release-15.

[4] D. Liu, W. Hong, T. S. Rappaport, C. Luxey, and W. Hong, "What will 5G Antennas and Propagation Be?," IEEE Trans. Antennas Propag., vol. 65, no. 12, pp. 6205-6212, Dec. 2017.

[5] P. Hindle, “Antenna Technologies for the Future,” Microw. J., vol. 61, no. 1, pp. 24-40, 2018.

[6] Shawn Knight, "Qualcomm announces first mmWave 5G antennas for mobile devices," TECHSPOT, 2018. [Online]. Available: https://www.techspot.com/news/75617qualcomm-announces-first-mmwave-5g-antennas-mobile-devices.html.

[7] R. W. Tkach, "Scaling optical communications for the next decade and beyond," Bell Labs Tech. J., vol. 14, no. 4, pp. 3-9, Feb. 2010.

[8] D. J. Richardson, J. M. Fini, and L. E. Nelson, "Space-division multiplexing in optical fibres," Nat. Photonics, vol. 7, no. 5, pp. 354-362, May 2013.

[9] B. Melián, M. Laguna, and J. A. Moreno-Pérez, "Capacity expansion of fiber optic networks with WDM systems: problem formulation and comparative analysis," Comput. Oper. Res., vol. 31, no. 3, pp. 461-472, Mar. 2004.

[10] E. Reyes-Vera, J. Úsuga, J. Acevedo-Echeverry, N. Gómez-Cardona, and M. Varón, 
"Performance analysis of a modal converter based on an asymmetric dual-core photonic crystal fiber," Opt. Pura y Apl., vol. 50, no. 3, pp. 251-257, Sep. 2017.

[11] S. Shwartz, M. Golub, and S. Ruschin, "Mode Multiplexer and Demultiplexer for SpaceDivision Multiplexing in Free Space and Fiber Optic Communication," in Asia Communications and Photonics Conference, 2013, p. AW3E.2.

[12] E. Agrell et al., "Roadmap of optical communications," J. Opt., vol. 18, no. 6, p. 063002, Jun. 2016.

[13] R. Martínez Ciro, F. López Giraldo, A. Betancur Perez, and M. Luna Rivera, "Characterization of Light-To-Frequency Converter for Visible Light Communication Systems," Electronics, vol. 7, no. 9, p. 165, Aug. 2018. 